\title{
Multi-user time-reversal STC-GFDMA for future wireless networks
}

\author{
Maximilian Matthe ${ }^{1 *}$, Luciano Leonel Mendes ${ }^{2}$, Ivan Gaspar ${ }^{1}$, Nicola Michailow ${ }^{1}$, Dan Zhang ${ }^{1}$ \\ and Gerhard Fettweis ${ }^{1}$
}

\begin{abstract}
The challenges of future wireless networks imply that the physical layer of the next generation mobile communication system needs to be compatible with multiple-input multiple-output and allow a flexible multiple access scheme. Time-reversal space-time coding can be applied to a recent filtered multicarrier modulation scheme, named generalized frequency division multiplexing, to achieve a multiple-input multiple-output non-orthogonal multicarrier modulation with flexibility to address the requirements of future mobile networks. In this paper, the subcarriers of the physical layer block are shared between multiple users, allowing for an efficient and simple multiple access solution. A channel estimation technique that can simultaneously estimate channel frequency response and timing misalignment in generalized frequency division multiple access is described. The paper also shows that the knowledge of channel state information can improve the overall performance when used to schedule the subchannel distribution among the users. Symbol error rate performance analysis shows that the resources of the time-reversal space-time coding generalized frequency division multiple access block can be shared among users that have one or two transmit antennas simultaneously under frequency-selective time-variant channels.
\end{abstract}

Keywords: Space-time coding; Multi-user access; Fading multipath channel; Generalized frequency division multiple access

\section{Introduction}

Next generation mobile communication systems will face new challenges that will require more than increasing the throughput [1]. Tactile Internet [2] demands for low latency. Multitude of devices will be connected in what is being called the Internet of Things [3]. Robustness will be required to deal with large coverage areas. Low out-ofband $(\mathrm{OOB})$ emission is mandatory for opportunistic use of vacant spectrum and fragmented spectrum allocation [4].

Several new multicarrier waveforms are being proposed to deal with these new requirements [1]. Generalized frequency division multiplexing (GFDM) [5] is one candidate that has the flexibility to address the upcoming demands of future networks. This modulation scheme employs $K$ individual circularly filtered subcarriers, each one carrying $M$ subsymbols of data. The circular filtering process

\footnotetext{
*Correspondence: maximilian.matthe@ifn.et.tu-dresden.de

1 Technische Universität Dresden, Georg-Schumann-Str. 11, D-01187 Dresden, Germany

Full list of author information is available at the end of the article
}

keeps the overall block length of $M K$ samples, where cyclic prefix and suffix can be applied to allow for simple frequency domain equalization [6] on the receiver side.

It is clear that any waveform for the next generation of mobile communication systems need to be compatible with multiple-input multiple-output (MIMO). Also, it needs to be easily combined with multiple access (MA) algorithms to share the physical layer (PHY) resources among several users and devices [1]. Recently, a simple approach for space-time coding GFDM has been published in [7]. However, the applied linear GFDM demodulator cannot decouple the subcarriers and subsymbols under the influence of multipath channels. Hence, when space-time coding (STC) is applied directly to the data symbols, residual multipath inter-symbol interference (ISI) leads to a severe performance loss, depending on the channel impulse response (CIR).

To overcome this problem, GFDM can be combined with time-reversal space-time coding (TR-STC) [8], to achieve full diversity gain while keeping the benefits of non-orthogonal waveforms [5]. With the application of

\section{Springer}

(c) 2015 Matthe et al: licensee Springer. This is an Open Access article distributed under the terms of the Creative Commons Attribution License (http://creativecommons.org/licenses/by/4.0), which permits unrestricted use, distribution, and reproduction in any medium, provided the original work is properly credited. 
TR-STC, the error floor due to multipath ISI is completely removed because space-time combining is performed in the signal domain rather than in the data domain. Also, the system complexity is kept low compared to more elaborate equalization techniques in the data domain [9].

The aim of this paper is to show that the resources of the TR-STC-GFDM can be shared among multiple users in the wireless up-link to achieve a TR-STC generalized frequency division multiple access (GFDMA) scheme. We show that the only requirement for this scheme is to have a band-guard subcarrier between the subchannels for each user. In fact, users with one or two transmit antennas can share the resources from the same TR-STC-GFDMA block. Also, we demonstrate that TR-STC-GFDMA tolerates timing misalignment between users with no penalty. Further, a scheduling mechanism to allocate users on certain subchannels based on their channel quality is presented, showing that the overall system performance can benefit from the knowledge of the channel state information (CSI). Additionally, the application of least-squares (LS) channel estimation for the presented multi-user scenario is contributed. Symbol error rate (SER) performance is simulated with perfect and estimated CSI, where we show that full diversity gain is achieved in both cases. The channel estimation error results in a constant performance loss; however, no other degradation is observed, showing that the channel and the timing misalignment between users can be successfully estimated and compensated with the proposed scheme.

The remainder of this paper is organized as follows: Section 2 presents the background of GFDM modulation, while Section 3 shows the principles of TR-STC. Section 4 presents GFDMA in combination with TR-STC. The performance of the proposed scheme is analyzed in Section 5 and conclusions are drawn in Section 6.

\section{GFDM Background}

GFDM is a block-based filtered multicarrier system where in each block $N=K M$ complex valued data symbols are distributed in time and frequency across $M$ subsymbols and $K$ subcarriers. $d_{k m}$ denotes the data symbol, taken from a $\mathcal{J}$-QAM constellation, that is to be transmitted on the $k$ th subcarrier and $m$ th subsymbol. Each $d_{k m}$ is transmitted with a specific waveform $g_{k m}[n]$ that is derived by a circular time-frequency shift of a prototype waveform $g[n]$ according to

$$
g_{k m}[n]=g\left[\langle n-m K\rangle_{N}\right] \exp \left(-j 2 \pi \frac{k n}{K}\right)
$$

where $n=0,1, \ldots, N-1$ is the time index of one GFDM block and $\langle\cdot\rangle_{N}$ is the modulo operator. $g[n]$ is typically a raised cosine (RC) filter with roll-off $\alpha$ [5], although other waveforms can be used. The transmit signal $x[n]$ of one GFDM block is the superposition of all constituting waveforms according to

$$
\vec{x}=\sum_{k=0}^{K-1} \sum_{m=0}^{M-1} \vec{g}_{k m} d_{k m}=\mathbf{A} \vec{d},
$$

where $\vec{x}$ is a column vector containing the samples of $x[n]$, $\vec{g}_{k m}$ is a column vector containing the samples of $g_{k m}[n]$, and $\vec{d}$ is a column vector containing $d_{k m}$ as its $(m K+k)$ th element. A is a $N \times N$ matrix, containing $\vec{g}_{k m}$ as its $(m K+$ $k)$ th column.

When $\vec{x}$ is transmitted over a multipath channel, with the help of a cyclic prefix (CP), the convolution with the (CIR) $h[n]$ becomes circular and hence low-complex zeroforcing (ZF) frequency domain equalization (FDE) can be carried out [5], leading to the equalized signal $\overrightarrow{\bar{y}}=\vec{x}+$ $\overrightarrow{\bar{w}}$ at the receiver, where $\bar{w}[n]$ is the colored noise due to channel equalization.

After equalization, convolution with a ZF receiver filter is carried out to detect the transmitted symbols. This operation can be expressed by

$$
\hat{\vec{d}}=\mathbf{B} \overrightarrow{\bar{y}}=\vec{d}+\mathbf{B} \overrightarrow{\vec{w}},
$$

where $\mathbf{B}=\mathbf{A}^{-1}$ and $\hat{\vec{d}}$ contains the estimated data symbols $\hat{d}_{k m}$ that are sent to the slicer. Note that $\mathbf{B}$ cancels self-interference occurring from the non-orthogonality of the transmit filter, but in turn introduces a noiseenhancement factor (NEF) $\xi$ given by

$$
\xi=\frac{1}{N} \operatorname{tr}\left(\mathbf{B}^{\mathrm{H}} \mathbf{B}\right) .
$$

\section{Time-reversal space-time coding}

TR-STC has been proposed by [8] to allow the use of STC for single carrier transmission over frequency-selective channels. The proposed approach operates on two subsequent data blocks $\vec{x}_{i}$ and $\vec{x}_{i+1}$ of length $N$ which are separated by a CP. Their corresponding discrete Fourier transforms are $\vec{X}_{(\cdot)}=\mathbf{F} \vec{x}_{(\cdot)}$, where $\mathbf{F}$ denotes the unitary Fourier matrix. The transmit signals on both antennas for two subsequent time slots are given by

\begin{tabular}{r|ll} 
& Antenna 1 & Antenna 2 \\
\hline Block $i$ & $\mathbf{F}^{\mathrm{H}} \vec{X}_{i}$ & $-\mathbf{F}^{\mathrm{H}} \vec{X}_{i+1}^{*}$ \\
Block $i+1$ & $\mathbf{F}^{\mathrm{H}} \vec{X}_{i+1}$ & $\mathbf{F}^{\mathrm{H}} \vec{X}_{i}^{*}$,
\end{tabular}

where $i$ is an even number and $(\cdot)^{\mathrm{H}}$ is the transposeconjugate operator. Note that the property

$$
\left(\mathbf{F}^{\mathrm{H}} \vec{X}_{i}^{*}\right)_{n}=x_{i}^{*}\left[\langle-n\rangle_{N}\right]
$$

of the discrete Fourier transform reasons the name 'timereversal space-time coding'. 
At the receiver, after removing the $\mathrm{CP}$, the transmit signals appear circularly convolved with the CIR $\vec{h}_{j, l}$, where $\vec{h}_{j, l}$ contains the channel taps between the $j$ th transmit and $l$ th receiving antenna, zero-padded to the block length. Both received blocks are transformed to the frequency domain. Accordingly, assuming the channel remains constant during the transmission of two subsequent blocks, the received blocks in the frequency domain are given by

$$
\begin{aligned}
\vec{Y}_{i, l} & =\mathbf{H}_{1, l} \vec{X}_{i}-\mathbf{H}_{2, l} \vec{X}_{i+1}^{*}+\vec{W}_{1, l} \\
\vec{Y}_{i+1, l} & =\mathbf{H}_{1, l} \vec{X}_{i+1}+\mathbf{H}_{2, l} \vec{X}_{i}^{*}+\vec{W}_{2, l}
\end{aligned}
$$

where $\mathbf{H}_{j, l}=\operatorname{diag}\left(\vec{H}_{j, i}\right)$ with $\vec{H}_{j, i}=\mathbf{F} \vec{h}_{j, l}$. The received signals can be combined in the frequency by

$$
\begin{aligned}
\hat{\vec{X}}_{i} & =\mathbf{H}_{e q}^{-1} \sum_{l=1}^{L} \mathbf{H}_{1, l}^{*} \vec{Y}_{i, l}+\mathbf{H}_{2, l} \vec{Y}_{i+1, l}^{*} \\
\hat{\vec{X}}_{i+1} & =\mathbf{H}_{e q}^{-1} \sum_{l=1}^{L} \mathbf{H}_{1, l}^{*} \vec{Y}_{i+1, l}-\mathbf{H}_{2, l} \vec{Y}_{i, l}^{*}, \\
\text { where } \mathbf{H}_{e q} & =\sum_{j=1}^{2} \sum_{l=1}^{L} \mathbf{H}_{j, l}^{*} \mathbf{H}_{j, l}
\end{aligned}
$$

and $L$ is the number of receiving antennas. Finally, the estimates of the transmitted blocks are acquired by inverse Fourier transform

$$
\hat{\vec{x}}_{i}=\mathbf{F}^{\mathrm{H}} \hat{\vec{X}}_{i}
$$

\section{Multi-user STC-GFDMA}

TR-STC can be directly applied to GFDM. Consider two data vectors $\vec{d}_{i}$ that generate two consecutive GFDM frames

$$
\vec{x}_{i}=\mathbf{A} \vec{d}_{i}
$$

The GFDM signals $\vec{x}_{i}$ and $\vec{x}_{i+1}$ can be space-time encoded as described in (5) and (9) can be used to recover the signals on the receiver side. Then, conventional GFDM demodulation with $\mathrm{ZF}$ receiver is carried out by

$$
\hat{\vec{d}}_{i}=\mathbf{B} \hat{\vec{x}}_{i}
$$

In this section, we combine TR-STC GFDM with a frequency division multiple access (FDMA) technique to serve multiple users with one GFDM system. The $K$ subcarriers are equally divided between $U$ users, i.e., each user allocates $K_{u}=K / U$ adjacent subcarriers. Further, resource allocation algorithms [10] can be used to distribute the subchannels between the users. Two situations are considered in this paper. First, we assume that CSI is not available at the base station to allow for subchannel scheduling and, second, CSI is considered to allocate the subchannels to the users.

Figure 1 depicts the block diagram of the proposed TRSTC-GFDMA system.

Each user $u$ generates a GFDM signal based on two successive data vectors $\vec{d}_{i}^{(u)}$, where elements corresponding to non-allocated subcarriers are set to zero. The data is modulated by $\mathbf{A}$ for each user and the blocks are spacetime encoded as described in (5). In order to combat time misalignment between users, in addition to the CP, a cyclic suffix (CS) is added to the blocks before they are transmitted through independent frequency-selective fading channels. When using a non-orthogonal transmit filter, adjacent subcarriers of two different users interfere with each other. Since the channels for the users are independent, these boundary subcarriers cannot be equalized and high inter-carrier-interference (ICI) occurs. Therefore, one guard subcarrier is used between users to avoid mutual interference. The guard subcarrier are unnecessary when an orthogonal pulse is used because, in this case, there is no ICI.

\subsection{Timing misalignment and channel estimation}

When all users are synchronized with the base station (BS) clock rate and all signals arrive within the CP and CS duration at the receive antennas, space-time combining according to (9) can be carried out per user, where only the users' allocated frequency samples are considered. However, CSI including misalignment information needs to be available at the BS. Note that timing misalignment within the $\mathrm{CP} / \mathrm{CS}$ length only results in a phase rotation of the circulant channel and hence channel equalization can compensate the misalignment. The CSI can be estimated at the BS by sending a separate pilot sequence per user that is transmitted twice in the TR-STC-GFDMA codeword. The sequences are modulated using an orthogonal pulse to avoid self-interference, with $K_{u}$ subcarriers per user and $M=1$ subsymbols. The signals are timereversal space-time encoded and CP and CS are appended before transmission.

At the BS, the received signal in the frequency domain is given by

$$
\begin{aligned}
\vec{P}_{i, l} & =\mathbf{P} \vec{H}_{1, l}-\mathbf{P}^{*} \vec{H}_{2, l}+\vec{W}_{i, l} \\
\vec{P}_{i+1, l} & =\mathbf{P} \vec{H}_{1, l}+\mathbf{P}^{*} \vec{H}_{2, l}+\vec{W}_{i+1, l},
\end{aligned}
$$

where $\mathbf{P}=\operatorname{diag}\left(\sum_{u=1}^{U} \vec{P}^{(u)}\right)$ with $\vec{P}^{(u)}$ containing the discrete Fourier transform (DFT) of the pilot sequence of the $u$ th user which is non-zero only at the allocated subcarriers and $\vec{H}_{j, l}=\left[\vec{H}_{j, l}^{(1)^{T}} \cdots \vec{H}_{j, l}^{(U)^{T}}\right]^{T}$ denotes the 


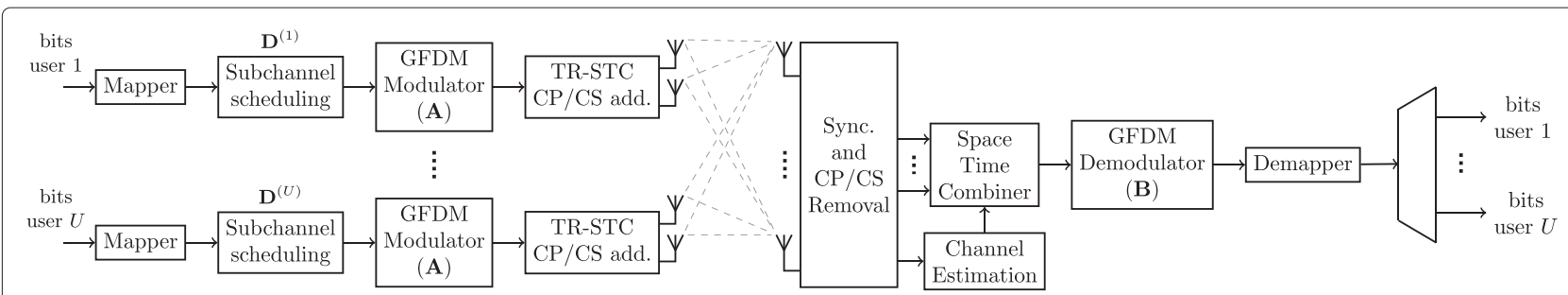

Figure 1 Simplified block diagram of the TR-STC-GFDMA system.

frequency response of the users' channels including time misalignment seen at the BS. Then, the CIR is estimated by

$$
\begin{aligned}
\vec{H}_{1, l} & =\frac{1}{2} \mathbf{P}^{-1}\left(\vec{P}_{i, l}+\vec{P}_{i+1, l}\right), \\
\vec{H}_{2, l} & =\frac{1}{2}\left(\mathbf{P}^{*}\right)^{-1}\left(\vec{P}_{i+1, l}-\vec{P}_{i, l}\right) .
\end{aligned}
$$

Note that the proposed scheme does not require all users to have two transmit antennas. Instead, using only the first transmit antenna is equivalent to have $\vec{h}_{2, l}=\overrightarrow{0}$. In this case, the transmitted signal can still be recovered; however, according to (9), no transmit diversity gain is achieved. For example, users with cheap devices or very good channel conditions would not carry out the spacetime encoding but can still be correctly received by the BS.

\subsection{User scheduling}

When it comes to multi-user scenarios, significant gains can be acquired by allocating each user to the subchannel that provides the best channel frequency response available. Let the available bandwidth be distributed into $U$ subchannels, where each subchannel contains $K / U$ subcarriers. Let the set of subcarriers of the $\kappa$ th subchannel for the $u$ th user be denoted by $\mathcal{K}_{u}$. Consider the scenario where time division duplexing (TDD) is employed and the $\mathrm{BS}$ is able to estimate the channel frequency response for each user. In this case, the BS can measure the quality $Q_{u, \kappa}$ of the $\kappa$ th subchannel of the $u$ th user as

$$
Q_{u, \kappa}=\sum_{k \in \mathcal{K}_{u}}{ }^{(u)} \mathbf{H}_{e q, k},
$$

where ${ }^{(u)} \mathbf{H}_{e q, k}$ is the $k$ th element on the diagonal of the equivalent channel of the $u$ th user, given by (10). Notice that the upper index $(u)$ has been used to denote the equivalent channel between the $u$ th user and the BS.

Based on $Q_{u, \kappa}$, the BS can schedule the best channel for the users using a priority-based scheduling algorithm. There, users are sorted according to their priorities and the user with highest priority receives the subchannel with highest quality. This subchannel is marked as unavailable for the other users and the procedure continues until the last user receives the last available subchannel.
In this paper, two simple approaches for the definition of the priority list are considered. In the first one, named 'random selection priority', the priorities of the users are randomly defined for each transmission, leading to a fair distribution between the users. For the second approach, named 'higher gain priority', the priority list is built in accordance with the channel quality from (18). In this case, the user that can experience the best channel condition is the first to receive a subchannel, which is marked as unavailable for the other users. The procedure repeats again until all subchannels are assigned to every user.

The main advantage of the higher gain priority over the random selection priority is that the former allows for the best use of the communication channel. However, higher gain priority reduces the chance of users with a single antenna to choose the subchannel first. Hence, when higher gain priority is employed, users with a single transmit antenna are expected to receive a smaller gain compared with the gain observed by users with two transmit antennas.

\section{Performance analysis}

The parameters used to evaluate the SER performance of the proposed system are presented in Table 1. The power delay profile of the wireless channel is shown in Table 2, which is based on the Extended Pedestrian A (EPA) model [11]. The channel taps are multiplied by i.i.d. complex Gaussian variables with variance $\sigma^{2}=1$,

\section{Table 1 Simulation parameters}

\begin{tabular}{lccc}
\hline Parameter & Symbol & GFDM & OFDM \\
\hline Mapper & $\mathcal{J}$-QAM & 16-QAM & 16-QAM \\
Transmit filter & $g[n]$ & RC, $\alpha=0.25$ & rect \\
\# Subcarriers & $K$ & 128 & 128 \\
\# Subsymbols & $M$ & 7 & 1 \\
CP length [samples] & $N_{C P}$ & 9 & 9 \\
CS length [samples] & $N_{C S}$ & 3 & 3 \\
NEF from (4) & $\xi$ & 1.02 & - \\
Overall NEF [dB] (23) & $\xi_{0}$ & 0.14 & 0.39 \\
\# Users & $U$ & 8 & 1 \\
\# Receiving antennas & $L$ & 1 & 1 \\
\hline
\end{tabular}


Table 2 Channel power delay profile used in the simulations

\begin{tabular}{lccccccc}
\hline Tap $(\boldsymbol{n}$ th sample) & $\mathbf{0}$ & $\mathbf{1}$ & $\mathbf{2}$ & $\mathbf{3}$ & $\mathbf{4}$ & $\mathbf{5}$ & $\mathbf{6}$ \\
\hline Tap gain $h_{n}[\mathrm{~dB}]$ & 0 & -1 & -2 & -3 & -8 & -17.2 & -20.8 \\
\hline
\end{tabular}

creating independent Rayleigh fading for each user and antenna. The channel remains constant during the transmission of two GFDM blocks. All users are synchronized with the base station's master clock, i.e., no frequency misalignment occurs. The timing misalignment between users and base station is uniformly distributed between $[-3,3]$ samples. Notice that the CP and CS are large enough to accommodate the channel impulse response and the time misalignment.

An approximation of the TR-STC-GFDM SER performance under a frequency-selective fading channel can be derived from an upper bound of symbol error probability for orthogonal maximum ratio combiner ([12], Ch. 13), but considering the NEF of GFDM. The approximation is given by

$$
p_{e} \approx 4 \mu \sum_{i=0}^{J L-1}\left(\begin{array}{c}
J L-1+i \\
i
\end{array}\right)\left(\frac{1+\eta}{2}\right)^{i},
$$

where

$$
\begin{aligned}
& \mu=\left(\frac{\sqrt{\mathcal{J}}-1}{\sqrt{\mathcal{J}}}\right)\left(\frac{1-\eta}{2}\right)^{J L}, \\
& \eta=\sqrt{\frac{\frac{3 \sigma_{e}^{2}}{\mathcal{J}-1} \frac{E_{s}}{\xi_{0} N_{0}}}{2+\frac{3 \sigma_{e}^{2}}{\mathcal{J}-1} \frac{E_{s}}{\xi_{0} N_{0}}}}
\end{aligned}
$$

with $E_{S}$ and $N_{0}$ denoting the average symbol energy and the noise power, respectively. $\mathcal{J}$ denotes the size of the digital constellation and

$$
\sigma_{e}^{2}=\sum_{n} \mathrm{E}\left[\left|h_{n}\right|^{2}\right]
$$

When considering the overhead of the CP of GFDM and orthogonal frequency division multiplexing (OFDM) in the effective noise calculation, we have

$$
\begin{aligned}
\xi_{0, \mathrm{OFDM}} & =\frac{K+N_{C P}+N_{C S}}{K} \\
\xi_{0, \mathrm{GFDM}} & =\xi \cdot \frac{K M+N_{C P}+N_{C S}}{K M},
\end{aligned}
$$

which shows that GFDM, depending on the NEF $\xi$ from (4), can achieve higher spectral efficiency compared to OFDM. This is due to the fact that GFDM requires only one CP for $M$ subsymbols, whereas OFDM uses one CP per OFDM symbol. As in [12], (19) becomes a tighter upper bound if $J L \geq 2$ and the channel frequency response is flat per subcarrier.

Figure 2 compares the SER performance of conventional STC-OFDM [13] and TR-STC-GFDMA with perfect CSI at the BS. It shows that TR-STC-GFDMA users with two transmit antennas (users 4 to 8 ) achieve the same diversity gain as STC-OFDM; however, due to the more efficient use of the CP, the SER curve of GFDM is shifted $0.25 \mathrm{~dB}$ to the left. As expected, users with one transmit antenna

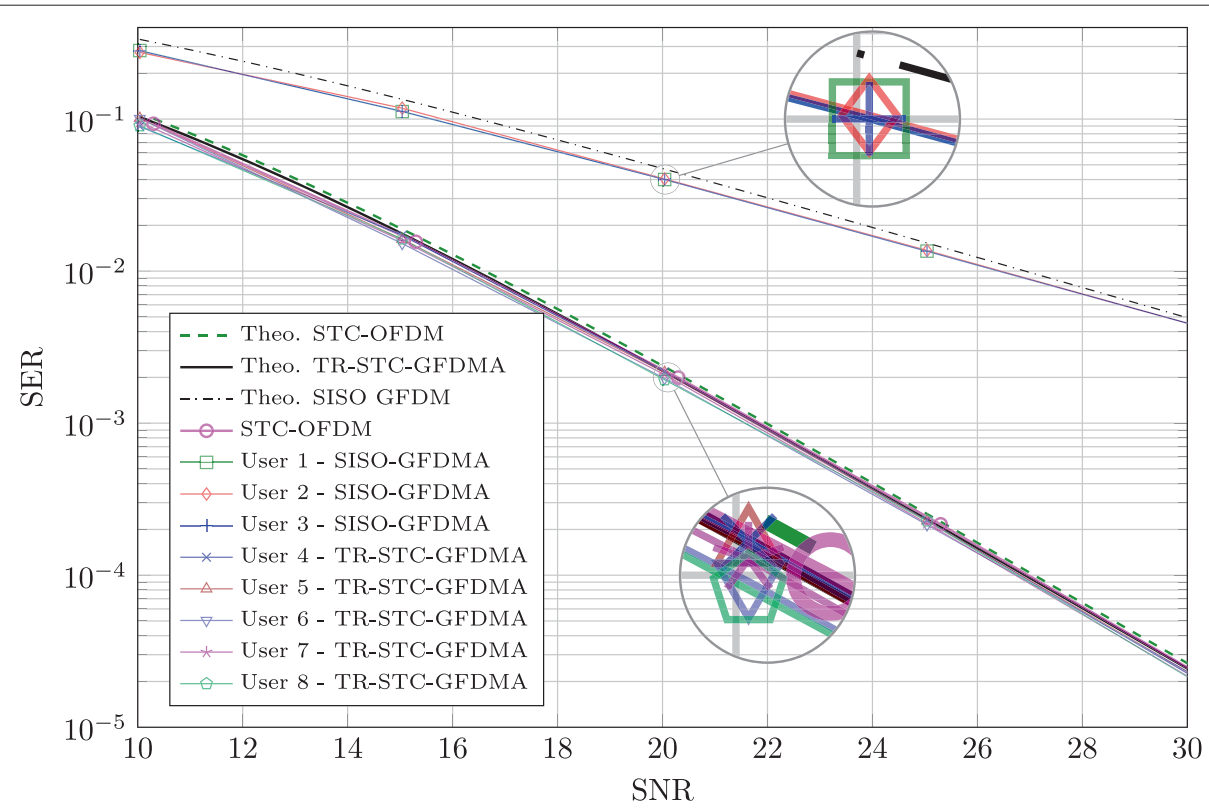

Figure 2 SER performance of STC-OFDM and TR-STC-GFDMA. 
(users 1 to 3 ) do not benefit from transmit diversity gain. Note that the benefit of employing GFDM here is not the SER performance gain compared with OFDM. As described in [5], GFDM has advantage of reducing the OOB emission, increasing the flexibility by covering other orthogonal waveforms as corner cases and better use of the time-frequency resource grid. Also, following (23), the TR-STC-GFDM SER performance can be improved by reducing the impact of the $\mathrm{CP}$ and $\mathrm{CS}$ in the overall overhead. This can be achieved by increasing the number of subsymbols.

Under the assumption of perfect CSI and knowledge of the time delay between users, it is evident that the TR-STC-GFDMA achieves full diversity. In a more realistic scenario, the BS needs to estimate the CSI and delay between users. However, if the time misalignment between users is smaller than the CP and CS length, the resulting effect is only the introduction of a phase rotation in the spectrum of the received signals. Under this condition, the BS only needs to estimate the overall equivalent channels for each user.

Figure 3 shows the SER performance with the proposed channel estimation algorithm. When a $3-\mathrm{dB}$ pilot boost is used, the performance is approximately $1.8 \mathrm{~dB}$ worse compared to perfect CSI but no further performance degradation in diversity is apparent.

The knowledge of the CSI at the BS side can improve the overall system performance by allocating the best available channel to a given user, in accordance with the channel quality presented in (18). Figure 4 presents the SER performance when random selection priority is employed to define the priority list. The performance gain for all users is clear. Users with single transmit antenna can observe a performance improvement of approximately 6 $\mathrm{dB}$, while users with two transmit antennas can benefit from an approximately 4-dB gain, when compared with the performance achieved with fixed subchannel allocation (Figure 2).

Figure 5 presents the achieved SER performance when the higher gain priority is used to build the priority list. As expected, users with two transmit antennas can benefit more from the subchannel allocation algorithm, resulting in a $5-\mathrm{dB}$ performance gain, compared to the fixed subchannel allocation. However, users with a single transmit antenna are more likely to have lower priority, meaning they have a smaller probability of choosing a high-quality subchannel. This results in a $3-\mathrm{dB}$ gain when compared with the performance achieved with fixed subchannel allocation.

\section{Conclusions}

A multiple access MIMO scheme is a mandatory requirement for the next generation of mobile communication systems. GFDM, which is a recent filtered multicarrier scheme, is flexible and can address the main requirements imposed by future networks. In this paper, it has been shown that GFDM can be easily integrated with TR-STC to achieve full diversity gain under frequencyselective fading channels. The paper also proposes an FDMA scheme, in order to share the subcarriers of the

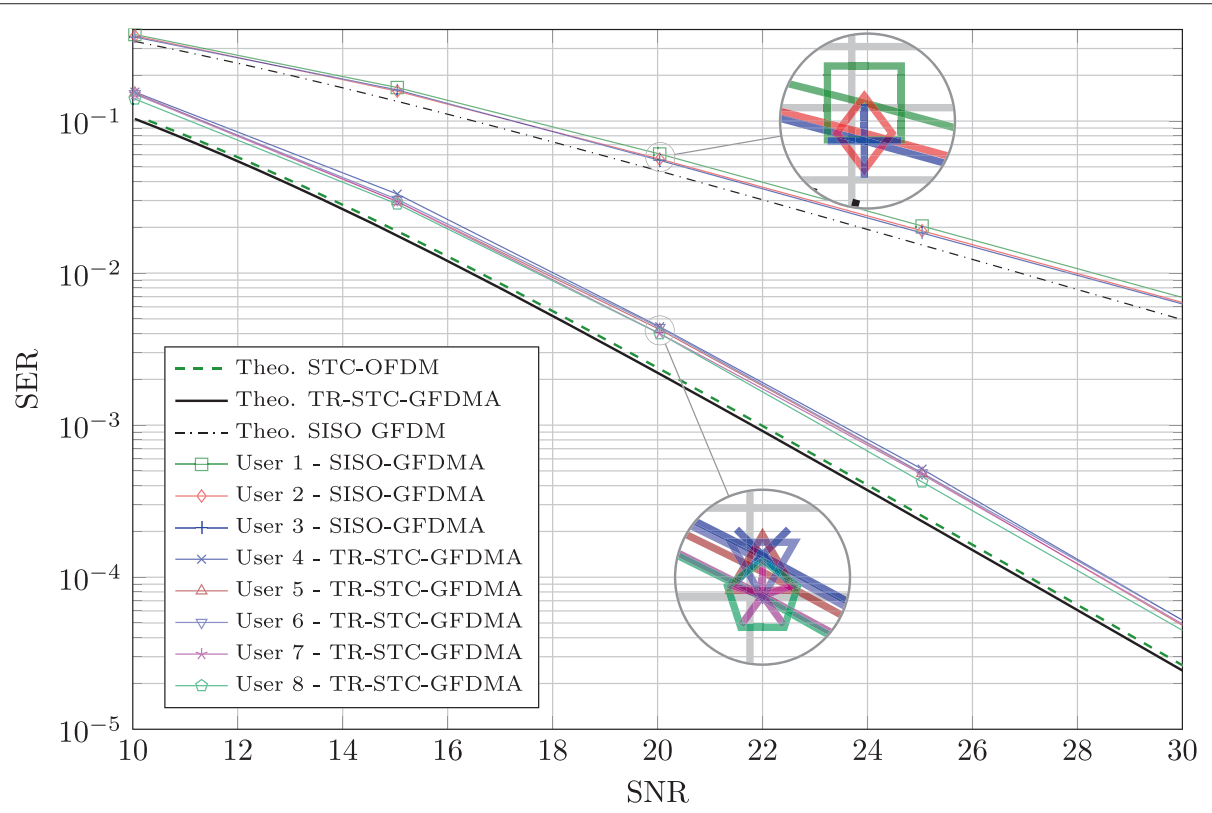

Figure 3 SER performance TR-STC-GFDMA considering the channel estimation. 


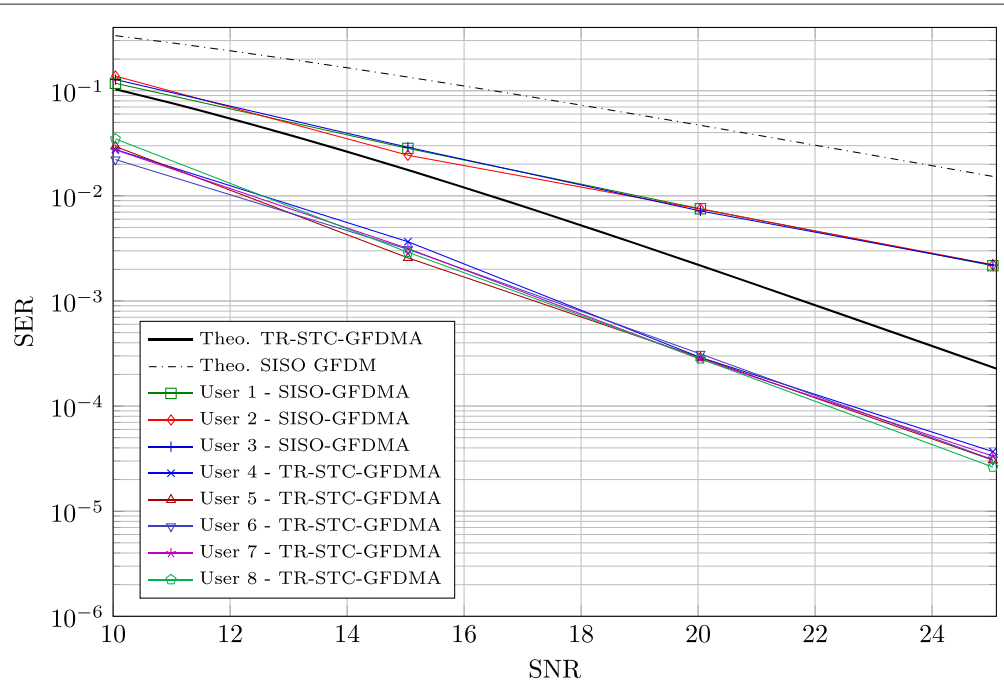

Figure 4 SER performance TR-STC-GFDMA assuming random selection priority.

TR-STC-GFDM signal among multiple users. Additionally, we present the application of a simple scheduling algorithm to distribute the available subchannels among the users. Two approaches have been considered, and the SER performance has been analyzed. The use of the CSI to assign the available subchannels to the users significantly improves the overall SER performance; however, the approach used to define the priority list impacts the observed gain. Timing misalignment between the signals received from multiple users can be compensated at the BS by a frequency domain equalizer when the overall misalignment and the channel length are within the $\mathrm{CP}$ and CS. When considering channel estimation, performance loss only occurs due to imperfect CSI at the BS, but no diversity loss can be observed. Finally, it is shown that the resources of the TR-STC-GFDMA block can be shared between users having one or two transmit antennas. Besides the loss of the diversity gain, no other consequence is observed for the users with only a single transmit antenna.

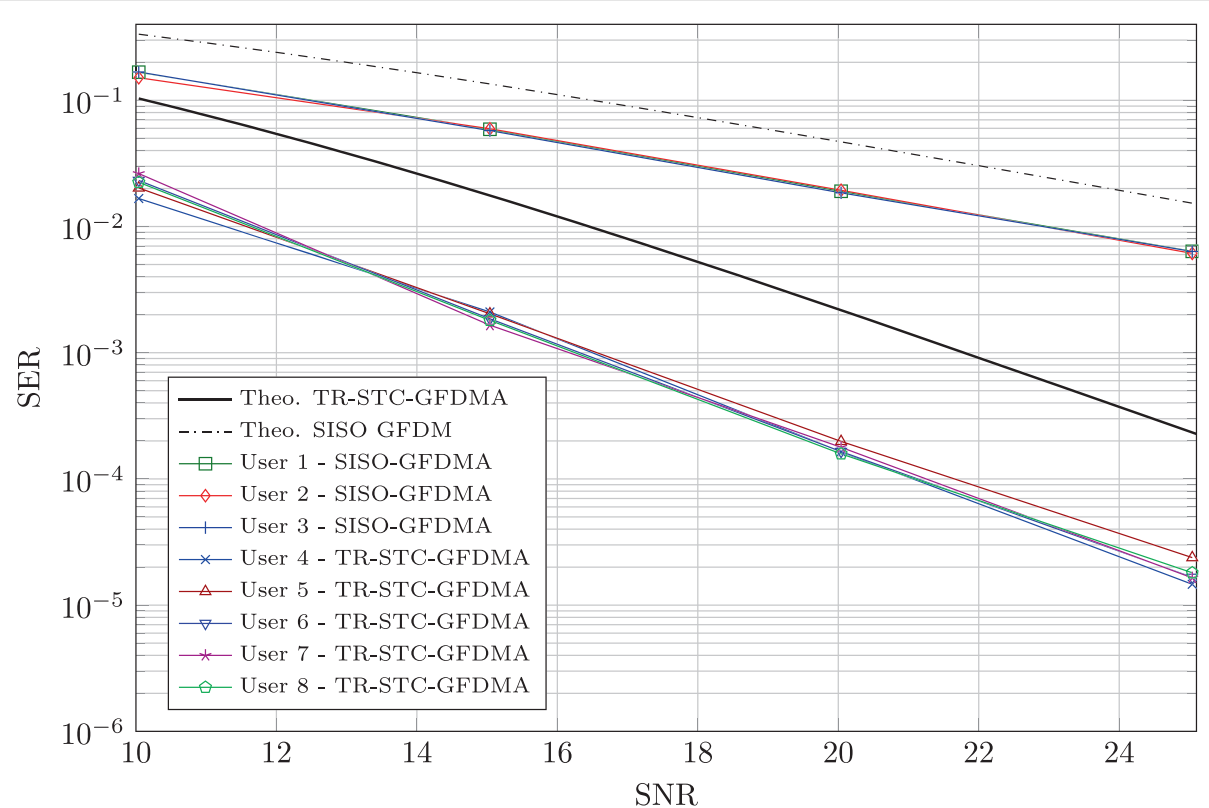

Figure 5 SER performance TR-STC-GFDMA assuming higher gain priority. 


\begin{abstract}
Abbreviations
$B S$, base station; CIR, channel impulse response; CP, cyclic prefix; CS, cyclic suffix; CSI, channel state information; DFT, discrete Fourier transform; EPA, Extended Pedestrian A; FDE, frequency domain equalization; FDMA, frequency division multiple access; GFDM, generalized frequency division multiplexing; GFDMA, generalized frequency division multiple access; ICI, inter-carrierinterference; ISI, inter-symbol interference; LS, least-squares; MA, multiple access; MIMO, multiple-input multiple-output; NEF, noise-enhancement factor; OFDM, orthogonal frequency sdivision multiplexing; OOB, out-of-band; PHY, physical layer; $R C$, raised cosine; SER, symbol error rate; SISO, single-input single-output; STC, space-time coding; TDD, time division duplexing; TR-STC, time-reversal space-time coding; ZF, zero-forcing.
\end{abstract}

\section{Competing interests}

The authors declare that they have no competing interests.

\section{Acknowledgements}

This work has been performed in the frameworks of the ICT project ICT-318555 '5GNOW' and FP7 ICT-619555 'RESCUE', which are partly funded by the European Union. The authors would like to thank Instituto Nacional de Telecomunicações (Inatel) and Conselho Nacional de Desenvolvimento Científico e Tecnológico (CNPq) for partially funding the work presented in this paper.

\section{Author details}

${ }^{1}$ Technische Universität Dresden, Georg-Schumann-Str. 11, D-01187 Dresden, Germany. ${ }^{2}$ Instituto Nacional de Telecomunicações, Av. João de Camargo, 510, 37540-000 Santa Rita do Sapucai, MG, Brazil.

Received: 12 December 2014 Accepted: 17 April 2015

Published online: 09 May 2015

\section{References}

1. G Wunder, P Jung, M Kasparick, T Wild, F Schaich, Y Chen, S Brink, I Gaspar, N Michailow, A Festag, L Mendes, N Cassiau, D Ktenas, M Dryjanski, S Pietrzyk, B Eged, P Vago, F Wiedmann, 5GNOW: non-orthogonal, asynchronous waveforms for future mobile applications. IEEE Commun. Mag. 52(2), 97-105 (2014)

2. GP Fettweis, The tactile internet: applications and challenges. IEEE Vehicular Technol. Mag. 9(1), 64-70 (2014)

3. $Y$ Ding, $Y$ Jin, $L$ Ren, $K$ Hao, An intelligent self-organization scheme for the internet of things. IEEE Comput. Intell. Mag. 8(3), 41-53 (2013)

4. $Y$ Zeng, $Y-C$ Liang, AT Hoang, $R$ Zhang, A review on spectrum sensing for cognitive radio: Challenges and solutions. EURASIP J. Adv. Signal Process. 2010, 1-16 (2010)

5. N Michailow, Matthé, I Gaspar, A Navarro Caldevilla, LL Mendes, A Festag, $G$ Fettweis, Generalized frequency division multiplexing for 5 th generation cellular networks. IEEE Trans. Commun. 62(9), 99 (2014)

6. D Falconer, SL Ariyavisitakul, A Benyamin-Seeyar, B Eidson, Frequency domain equalization for single-carrier broadband wireless systems. IEEE Commun. Mag. 40(4), 58-66 (2002)

7. Matthé, LL Mendes, G Fettweis, in European Wireless 2014 (EW2014). Space-time coding for generalized frequency division multiplexing (VDE, Barcelona, Spain, 2014)

8. N Al-Dhahir, Single-carrier frequency-domain equalization for space-time block-coded transmissions over frequency-selective fading channels. IEEE Commun. Lett. 5(7), 304-306 (2001)

9. WH Gerstacker, F Obernosterer, R Schober, A Lehmann, A Lampe, P Gunreben, in Proceedings IEEE 56th Vehicular Technology Conference. Widely linear equalization for space-time block-coded transmission over fading ISI channels, vol. 1 (IEEE, 2002), pp. 238-242. doi: 10.1109/NETECF.2002.1040340

10. VD Papoutsis, SA Kotsopoulos, in ICWMC 2011 : The Seventh International Conference on Wireless and Mobile Communications. Efficient rate adaptive resource allocation scheme in uplink OFDMA wireless systems (IARIA Luxembourg, 2011)

11. F Rezaei, M Hempel, H Sharif, in Proc. IEEE 16th International Workshop on Computer Aided Modeling and Design of Communication Links and Networks (CAMAD). LTE PHY performance analysis under 3GPP standards parameters (IEEE Kyoto, 2011), pp. 102-106
12. S Benedetto, E Biglieri, Principles of Digital Transmission with Wireless Applications. (Kluwer Academic/Plenum Press, Norwell, MA, USA, 1999)

13. K Lee, D Williams, in Proceedings of the IEEE Sensor Array and Multichannel Signal Processing Workshop. A space-time coded transmitter diversity technique for frequency selective fading channels (IEEE Cambridge, 2000), pp. 149-152. doi: 10.1109/SAM.2000.877987

\section{Submit your manuscript to a SpringerOpen ${ }^{\mathcal{O}}$ journal and benefit from:}

- Convenient online submission

- Rigorous peer review

- Immediate publication on acceptance

- Open access: articles freely available online

- High visibility within the field

- Retaining the copyright to your article

Submit your next manuscript at $>$ springeropen.com 